\title{
Overcoming Filtering Penalties in Flexi-Grid Long-Haul Optical Systems
}

\author{
Tommaso Foggi, Giulio Colavolpe Senior Member, IEEE, Alberto Bononi Senior Member, IEEE, and Paolo \\ Serena Member, IEEE
}

\begin{abstract}
Flexible grid optical networks allow a better exploitation of fiber capacity, by enabling a denser frequency allocation. A tighter channel spacing, however, requires narrower filters, which increase linear intersymbol interference (ISI), and may dramatically reduce system reach. Commercial coherent receivers are based on symbol by symbol detectors, which are quite sensitive to ISI. In this context, Nyquist spacing is considered as the ultimate limit to wavelength-division multiplexing (WDM) packing.

In this paper, we show that by introducing a limited-complexity trellis processing in the receiver, either the reach of Nyquist WDM flexi-grid networks can be significantly extended, or a higher spectral efficiency (SE) is possible at equal reach. By adopting information-theoretic techniques, we design a limitedcomplexity trellis processing and quantify its SE gain in flexigrid architectures where wavelength selective switches over a frequency grid with $12.5 \mathrm{GHz}$ granularity are employed.
\end{abstract}

Index Terms-Optical communications, Coherent detection, Nonlinear propagation, Nyquist-WDM, Time-frequency packing, Polarization-multiplexed quaternary phase-shift keying, Flexigrid, ROADM.

\section{INTRODUCTION}

Spurred by the relentless increase of data traffic, coherent optical systems were revived in the last decade, and many different paths were undertaken in order to better exploit the huge capacity of the fiber channel, from polarization-multiplexing (PM) and high-order modulations, to dense wavelength division multiplexing (WDM) solutions like Nyquist-WDM or orthogonal frequency division multipleximg (OFDM) [1], [2]. Although to cope with the foreseen capacity crunch of the existing fiber infrastructure [3] the long-term solution will likely be the use of multimode fibers and multi-input-multi-output processing-a solution that requires replacing the existing fiber infrastructure-yet a less disruptive step towards increasing SE has been the introduction of flexi-grid WDM networks [4], where throughput increase is achieved through the reduction of channel spacing. However, the deployment of new generation flexible wavelength selective switches (WSS) [5] in reconfigurable optical add-and-drop multiplexers (ROADM), compatible with the aforementioned standard, entails a careful redesign of optical systems, and specifically transmission and reception techniques. In fact, the presence of WSS with 12.5 GHz granularity prevents the SE increase through the simple adoption of Nyquist-shaped signaling, as the effect of crossing ROADMs, and therefore cascaded WSS filtering, is detrimental even after a few nodes [6].

We employ a maximum-a-posteriori probability (MAP) symbol detector [7] with a minimum number of states in

T. Foggi is with CNIT Research Unit, I-43124 Parma, Italy. G. Colavolpe A. Bononi and P. Serena are with Dip. Ing. Informazione, Università degli Studi di Parma, I-43124 Parma, Italy. order to mitigate the effects of WSS inline filtering, and compare it to the conventional symbol-by-symbol ( $\mathrm{SbS})$ detector. The comparison is carried out employing a polarizationmultiplexed quaternary phase shift keying (QPSK) modulation, both in terms of pre forward-error-correction (FEC) bit error rate (or equivalently Q-factor) and in terms of achievable information rate (AIR), and thus achievable SE, by resorting to the simulation-based technique detailed in [8]. While the AIR represents a theoretical value that may be achieved by some optimal FEC, we next extend the analysis by equipping our receivers with existing low-density parity check codes (LDPC) designed for satellite links [9], and check their SE against that obtained from AIR. The SE of such practical LDPCs is close to the SE forecast by the AIR. We prove that even a 2 -state MAP symbol detector ${ }^{1}$ allows, for instance, to more than double the maximum reach when using $25 \%$ overhead FECs and a Nyquist channel spacing. There are other techniques based on advanced signal processing (e.g., see [10]-[13]), though our work for the first time presents results for a flexi-grid system heavily impaired by tight inline filtering, and exploiting an information theoretic analysis to design the receiver processing.

The paper is organized as follows. In Section II, a description of the system model link architecture and the adopted detection strategy are provided. Section III gives insights on the theoretical approach chosen to evaluate the receiver performance and to provide an analysis of system impairments. Section IV reports the numerical results and the observations that arise. Finally, conclusions are drawn in Section V.

\section{SYSTEM MODEL}

The considered system is closely related to that described in [6]. In our WDM simulated system, the carriers of the $N_{c}$ PM linearly-modulated signals have a random polarization and random detuning with respect to their nominal frequencies.. In the following, we will consider a QPSK modulation format on each carrier and each polarization. The general expression of the complex envelope of the signal transmitted on the $\ell$ th carrier and the $i$ th polarization $(i=1,2)$ is

$$
\sum_{k=0}^{K-1} x_{k}^{(i, \ell)} p\left(t-k T-\tau^{(i, \ell)}\right) e^{j\left[2 \pi \ell\left(F+\Delta_{\ell}\right) t+\theta^{(i, \ell)}\right]}
$$

where $p(t)$ is the shaping pulse having root raised-cosine (RRC) spectrum with roll-off $\alpha=0.1$ (obtained through the use of a proper transmit-side electrical filter), $K$ the number of symbols transmitted over each carrier and each polarization,

\footnotetext{
${ }^{1}$ Notice that our receiver processes the PM-QPSK signal components separately, thus it entails four 2-state MAP detectors.
} 
$T$ the symbol interval, $x_{k}^{(i, \ell)}$ the symbol transmitted over the $\ell$ th carrier of the $i$ th polarization during the $k$ th symbol interval, $\tau^{(i, \ell)}$ and $\theta^{(i, \ell)}$ the delay and the initial phase of the $i$ th polarization and $\ell$ th carrier, respectively, $F$ the frequency spacing between two adjacent carriers, and $\Delta_{\ell}$ a possible frequency offset (small compared to the frequency spacing). The transmitted symbols were obtained from a stream of information bits, properly encoded through a binary channel $\operatorname{code}^{2}$ and Gray mapped onto the QPSK constellation.

The transmitted signal was then launched into a dispersion unmanaged fiber link with variable number of spans $N_{s}$, characterized by the presence of ROADM nodes, one every two fiber spans. Therefore, the number of crossed ROADMs was equal to half the number of spans. Each span had 120 $\mathrm{km}$ of single mode fiber (SMF) and an erbium-doped fiber amplifier (EDFA) with a noise figure of $6 \mathrm{~dB}$. Since ROADMs are here intended as simple pass-through nodes, they basically just introduce the filtering effect of two WSS, modeled as 3rdorder Gaussian filters. The bandwidth of such filters can be determined once the flexible grid spacing has been selected. We fixed the channel spacing to $37.5 \mathrm{GHz}$, which implies a 3-dB filter bandwidth of $35.75 \mathrm{GHz}$ [5], [6].

Fiber propagation was impaired by group velocity dispersion (GVD), ${ }^{3}$ and nonlinear effects. These latter effects were simulated by the split step Fourier method (SSFM) [14] applied to the Manakov nonlinear equation with proper step size. ${ }^{4}$ The symbol rate $R$ of each signal was fixed to 32.5 Gbaud, as in [6]. Fig. 1 shows the block diagram of the generic simulated link.

At the receive side, coherent detection was performed (as in [15]). The received optical field was first filtered by a 4th-order super-Gaussian filter having a 3-dB bandwidth of $35.75 \mathrm{GHz}$, which allows to select the desired channel, and then converted to the electrical domain through a $90^{\circ}$ optical hybrid. Digital signal processing (DSP) was then performed, as explained in detail in [8]. After sampling, compensation for the cumulated GVD was performed by two fixed-tap equalizers (one per polarization) and then frame and frequency synchronization and compensation were performed. A two-dimensional fractionally-spaced adaptive minimum mean square error (MMSE) feed-forward equalizer (FFE) performed compensation of the residual GVD and polarization mode dispersion (PMD), and also performed polarization demultiplexing. The number of taps was chosen sufficiently high so that linear impairments did not entail any penalty, whereas a coefficient adaptation step-size value of $10^{-3}$ came up to be optimal in any case. Notice that the equalizer purpose is the compensation of the impairments not envisaged in the auxiliary channel. Finally phase noise was tracked by a proper phase estimation and compensation module (see [16]). All synchronization aspects were neglected here-perfect synchronization was assumed. These aspects will be discussed in detail

\footnotetext{
${ }^{2}$ Binary LDPC coding is considered in the numerical results.

${ }^{3}$ We also considered polarization mode dispersion (PMD) with values of the differential group delay of typical fibers and noted no performance difference. Thus, PMD is not present in current results.

${ }^{4}$ We optimized the step-size for each launch power, increasing the value in trial simulations until we noted no performance variation.
}

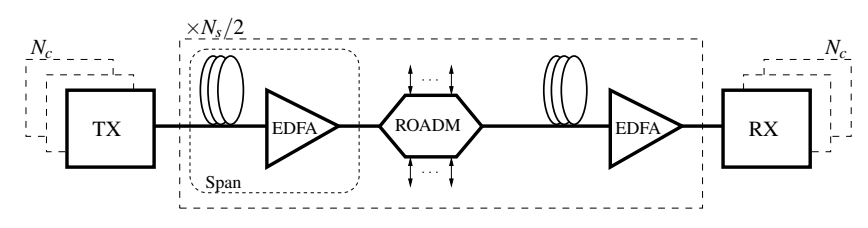

Figure 1. Schematic of the simulated optical link.

in a future paper. Finally, signal samples fed the detector, which, in the MAP case, iteratively exchanges soft information with the LDPC decoder.

We considered two kinds of detectors. First, we used a conventional symbol-by-symbol detector/demapper that neglects channel memory (i.e., the detector commonly used in coherent receivers). As a second more sophisticated solution, we employed a MAP symbol detector with a minimum number of states (see Section IV), preceeded by a channel shortener [17], which is essentially a linear filter of a few taps, and whose computation is based on the estimation of the overall channel impulse response. The shortener helps coping with the intersymbol interference (ISI) not accounted for by the limited detector memory, as explained in [8]. More details on the simulated system and receiver parameters will be given in Section IV.

On the described system, we performed two kinds of simulations. First, we evaluated the achievable SE, as explained in the next section, by varying the launch power per channel and number of spans $N_{s}$ of the link. Then, we also employed a set of codes with rates in the range 1/3-9/10. These codes were used to confirm the AIR predictions.

\section{Spectral EfFiciency Analysis}

We now describe the framework used to evaluate the performance limits of all the optical transmission systems considered in this paper.

We consider an optical channel with linear and nonlinear distortions, simulated through the SSFM. Denoting by y the suitably processed sequence of the received channel time samples which we use for detection of the information symbols $\mathbf{x}=\left\{x_{k}^{(i, \ell)}\right\}_{k, i, \ell}$, the information rate (IR), i.e., the average mutual information when the information symbols are independent and uniformly distributed random variables belonging to the given constellation, is defined as ${ }^{5}$

$I(\mathbf{x} ; \mathbf{y})=\lim _{K \rightarrow \infty} \frac{1}{2 N_{c} K} E\left\{\log _{2} \frac{p(\mathbf{y} \mid \mathbf{x})}{\sum_{\mathbf{x}^{\prime}} p\left(\mathbf{y} \mid \mathbf{x}^{\prime}\right) P_{m}\left(\mathbf{x}^{\prime}\right)}\right\}\left[\frac{\text { bit }}{\text { ch. use }}\right]$

where $E[\cdot]$ denotes expectation, $p(\cdot)$ a probability density function (PDF) and $P_{m}(\cdot)$ a probability mass function (PMF). The SE is the IR per unit bandwidth and unit time

$$
\eta=\frac{I(\mathbf{x} ; \mathbf{y})}{F T} \quad[\mathrm{~b} / \mathrm{s} / \mathrm{Hz}]
$$

since $F T$ is the time-frequency slot devoted to the transmission of symbol $x_{k}^{(i, \ell)}$.

\footnotetext{
${ }^{5}$ The factor 2 , takes into account the presence of two polarizations.
} 
The computation of IR and SE requires the availability of the pdfs $p(\mathbf{y} \mid \mathbf{x})$ and $p(\mathbf{y})=\sum_{\mathbf{x}^{\prime}} p\left(\mathbf{y} \mid \mathbf{x}^{\prime}\right) P_{m}\left(\mathbf{x}^{\prime}\right)$. However, they are not known in closed form, nor can we resort to the simulation method in [18] to compute them. In fact, this method requires that the channel at hand has a finite memory and the availability of an optimal detector for it [18]. These conditions are clearly not satisfied in our scenario [19], [20]. We may thus resort to the computation of a proper lower bound of the IR (and thus of the SE) obtained by substituting $p(\mathbf{y} \mid \mathbf{x})$ in (2) with an arbitrary auxiliary channel law $q(\mathbf{y} \mid \mathbf{x})$ with the same input and output alphabets as the original channel (mismatched detection [18], [21]-[23]). The resulting lower bound reads as

$$
I_{\mathrm{LB}}(\mathbf{x} ; \mathbf{y})=\lim _{K \rightarrow \infty} \frac{1}{2 N_{c} K} E\left\{\log _{2} \frac{q(\mathbf{y} \mid \mathbf{x})}{\sum_{\mathbf{x}^{\prime}} q\left(\mathbf{y} \mid \mathbf{x}^{\prime}\right) P_{m}\left(\mathbf{x}^{\prime}\right)}\right\} .
$$

If the auxiliary channel law is representative of a finite-state channel, pdfs $q(\mathbf{y} \mid \mathbf{x})$ and $q_{p}(\mathbf{y})=\sum_{\mathbf{x}^{\prime}} q\left(\mathbf{y} \mid \mathbf{x}^{\prime}\right) P_{m}\left(\mathbf{x}^{\prime}\right)$ can be computed, this time, by using the optimal MAP symbol detector for that auxiliary channel [18]. Such detector, that will clearly be suboptimal for the actual channel, will have at its input the sequence $\mathbf{y}$ generated by simulation according to the actual channel model, and the expectation in (3) is meant with respect to the input and output sequences generated accordingly [18]. Thus, no assumption on the true statistics of the discrete-time received sequence is required for the design of the adopted detector, since it is designed for the auxiliary channel. Similarly, the true statistics of the sequence $\mathbf{y}$ are not analytically required for its generation, since they can be obtained by SSFM simulation through the actual nonlinear channel. If we change the adopted receiver (or, equivalently, if we change the auxiliary channel based on which its trellis metrics are expressed) we obtain different lower bounds on the information rate but, in any case, such bounds are achievable by those receivers, according to mismatched detection [18],[21]. We will thus say, with an abuse of terminology, that the computed lower bounds are the SE values of the considered channel when those receivers are employed. All these considerations hold for any actual channel, including nonlinear and non-Gaussian ones.

This technique thus allows to evaluate the AIR for receivers of reduced complexity. In fact, it is sufficient to consider an auxiliary channel which is a simplified version of the actual channel in the sense that only a portion of the true channel memory and/or a limited number of impairments are present. The considered receivers have been described in the previous section. As mentioned, we have assumed that parallel independent detectors are employed, one for each carrier and each polarization. In other words, intercarrier interference (ICI) is not coped with at the receiver, since multiuser detection is considered too computationally demanding. This corresponds to the adoption of an auxiliary channel model that can be factorized into the product

$$
q(\mathbf{y} \mid \mathbf{x})=\prod_{i} \prod_{\ell} q\left(\mathbf{y}^{(i, \ell)} \mid \mathbf{x}^{(i, \ell)}\right)
$$

where $\mathbf{y}^{(i, \ell)}$ is a proper discrete-time received sequence used for detection of symbols $\mathbf{x}^{(i, \ell)}=\left\{x_{k}^{(i, \ell)}\right\}$ transmitted over the $\ell$ th carrier and the $i$ th polarization. Under this hypothesis, we simply have

$$
I_{\mathrm{LB}}(\mathbf{x} ; \mathbf{y})=\lim _{K \rightarrow \infty} \frac{1}{K} E\left\{\log _{2} \frac{q\left(\mathbf{y}^{(i, \ell)} \mid \mathbf{x}^{(i, \ell)}\right)}{q_{p}\left(\mathbf{y}^{(i, \ell)}\right)}\right\},
$$

i.e., the result can be computed by considering only one carrier. In a practical scenario with a finite number of carriers, we will consider the central carrier only, avoiding the computation on the border carriers which are affected by a smaller amount of ICI, thus obtaining a further lower bound.

Note that, as stated, we are not able to compute the IR of the true channel since the optimal receiver is unknown and possibly of unmanageable complexity. Nevertheless, by the auxiliary channel method we get an achievable IR, i.e., there exist a code reaching such a target. We take there the pragmatic approach of considering only limited-complexity suboptimal receivers. For such receivers we are indeed able to compute the relevant IR which will be called achievable IR. The corresponding achievable lower bound on SE (achievable $\mathrm{SE}$ in the following) is thus

$$
\eta_{\mathrm{LB}}=\frac{1}{F T} I_{\mathrm{LB}}(\mathbf{x} ; \mathbf{y}) \quad[\mathrm{b} / \mathrm{s} / \mathrm{Hz}] .
$$

The auxiliary channel that we adopted for the MAP symbol detector design neglects the presence of channel nonlinear effects, and assumes that GVD and PMD have been perfectly compensated. Basically, the detector is designed by taking into account transmit side shaping pulse and inline filtering, i.e., the sufficient statistics $\mathbf{y}^{(i, \ell)}$ can be obtained by sampling the output of a filter matched to the received pulse in the absence of GVD, PMD, and nonlinear effects, i.e., the transmit pulse after cascaded inline filtering. ${ }^{6}$ In SSFM simulations, noise contributions introduced by EDFAs are added along the whole optical link at each span. Hence inline filtering has an incremental effect on the propagating signal, whereas for the auxiliary channel we assume that all noise is added at the end of the link. Thus, at the receive side it is possible to estimate the overall channel response (through a simple MMSE estimator) without any knowledge of the link configuration, which corresponds to the most practical way to design the MAP symbol detector. Notice that the presence of other conventional receive-side filters, with bandwidth compatible with the chosen frequency grid, does not imply relevant changes to the established matched filter response, thus does not affect the aforementioned considerations. Given this auxiliary channel law, the optimal MAP symbol detector is described in [24] (see [8] for more details).

As a concluding remark, we would like to point out that this technique allows to compute the achievable limit of the

\footnotetext{
${ }^{6}$ The FFE taps are designed using the matched filter output as target response, so that the equalizer does not remove the ISI induced by filtering but only performs matched filtering. It is worth noting that, if extremely narrow optical filtering is employed at the receive side, the electrical compensation of chromatic dispersion through the non-adaptive equalizers may result to be inaccurate. In this case, a wider optical filter can be used, compatibly with the system design, in order to leave the useful component of the received signal unchanged, whereas matched filtering is implemented by the adaptive equalizer.
} 


\begin{tabular}{|c|c|c|c|c|}
\hline & TX Optical & Inline WSS & RX Optical & RX electrical \\
\hline Type & RRC, $\alpha=0.1$ & 3rd Gauss. & 4th Gauss. & 5th Bessel \\
\hline Bandwidth [GHz] & 32.5 & 35.75 & 35.75 & 16 \\
\hline
\end{tabular}

Table I

FILTERS PARAMETERS

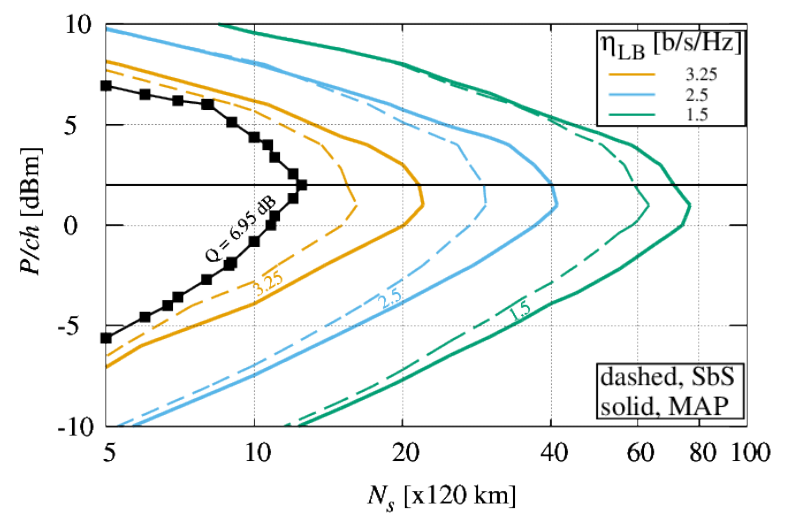

Figure 2. Horizontal cuts of the spectral efficiency as a function of the launch power per channel and number of spans, $R=32.5$ Gbaud, $F=37.5 \mathrm{GHz}$, MAP detector with $L=1$ and conventional symbol-by-symbol detector. It is also reported the horizontal cut corresponding to $\mathrm{BER}=0.0135$, or $\mathrm{Q}$-factor $=6.9$ $\mathrm{dB}$, assumed as a pre-FEC threshold for $25 \%$ overhead code, symbol-bysymbol detector.

considered receivers without taking into account specific coding schemes, being understood that, with a properly designed channel code, the information-theoretic performance can be closely approached. Section IV will report some design cases for these codes with the aim of showing that, indeed, the performance predicted by the achievable SE can be approached.

\section{NUMERICAL RESULTS}

In this section, we present the simulation results of the optical channel described in Section II. The WDM input signal in (1) has 11 channels with spacing $37.5 \mathrm{GHz}$ and was launched in the line with $P /$ ch power per channel and propagated through a dispersion-uncompensated line of $N_{s}$ identical 120 $\mathrm{km}$ single-mode fiber (SMF) spans. Fiber parameters include dispersion $16.63 \mathrm{ps} / \mathrm{nm} / \mathrm{km}$, attenuation of $0.23 \mathrm{~dB} / \mathrm{km}$, and nonlinear index $\gamma$ equal to $1.3 \mathrm{~W}^{-1} \mathrm{~km}^{-1}$. Every two spans we included a ROADM, whose only effect is a filtering due to the presence of two WSS on the signal path. In Tab. I, we recall system filter types and bandwidths, which are kept unchanged in all simulations. The effect of cascaded WSS is then summarized in Tab. II, which reports the concatenated bandwidth $(\mathrm{CBW})$, versus the number of crossed WSS.

We simulated the propagation of $R=32.5$ Gbaud channels, and each of them was detected by using proper MAP symbol detectors which take into account a memory of $L=1$ symbols. Since we use a QPSK modulation per polarization, the detector was split into 4 binary detectors with $2^{L}=2$ states, each operating on one polarization and one quadrature. In addition, we also considered the use of a standard symbol-by-symbol detector. Results are shown in Fig. 2, where horizontal cuts of SE surfaces are plotted. For each point of the surface we averaged over 6 clusters of about 70000 symbols, simulated using a pseudo-random bit generator, obtaining a confidence interval of at worst $4 \%$. Furthermore, for each point the

\begin{tabular}{|c|c|c|c|c|c|c|c|}
\hline \# WSS & 2 & 4 & 8 & 12 & 16 & 32 & 64 \\
\hline 3-dB CBW [GHz] & 31.85 & 28.4 & 25.2 & 23.6 & 22.5 & 20 & 17.8 \\
Table II \\
CONCATENATED BANDWIDTH VS. CROSSED WSS.
\end{tabular}

transmitted channels were launched with random initial polarizations, delays and offset frequencies. As far as the frequency offset is concerned, it can be considered to be two orders of magnitude smaller than the carrier spacing. In our simulations we included random offsets in the range $\pm 1 \%$ of the carrier spacing. This choice is not dictated by the need to perform carrier synchronization. In fact, plenty of algorithms able to recover a frequency offset up to $30-40 \%$ of the symbol rate are available in the literature (e.g., see [25] and references therein). On the other hand, with the tight filters used in flexi-grid systems, a larger frequency offset would have a negative impact on the signal power at the filters' output. So a more strict control on the frequency instabilities must be adopted with respect to classical systems. Nevertheless, we also performed a few simulations with frequency offsets up to $\pm 5 \%$ of the carrier spacing, observing negligible penalty. The smoothness of the resulting surfaces confirms the validity of this averaging choice. We also report in this figure the horizontal cut corresponding to a bit error rate (BER) of $\sim 0.0132$ (or, equivalently, Q-factor equal to $6.95 \mathrm{~dB}$ ) for the symbol-by-symbol detector, which represents a conventional pre-FEC BER for a $25 \%$ overhead code (to be more precise, a concatenated $\mathrm{BCH}$ code, see [26]). From the figures, it can be noticed that the theoretical SE $\eta_{\mathrm{LB}}$ (back-to-back case) of $\sim 4 \cdot R / F[\mathrm{~b} / \mathrm{s} / \mathrm{Hz}]$ decreases with increasing distance, and MAP detector shows a clear maximum reach advantage.

Fig. 3 shows instead the vertical cuts of the SE surfaces at $P / c h=2 \mathrm{dBm}$. For this scenario, we also simulated coded signaling, using LDPC codes with rates from $1 / 3$ to $9 / 10$ from [9], setting the maximum reach at distances where a bit error rate lower than $10^{-4}$ is achieved, which actually means convergence of the iterative detection/decoding algorithm ${ }^{7}$. Notice that we used available LDPC codes known from literature for our simulations, nevertheless these codes are designed for the AWGN channel, hence better results can be expected with a careful ad hoc design of proper codes. We fixed a limit of 40 iterations, and averaged over 500,000 received symbols per step. We found a good agreement between expected results from achievable lower bounds and simulations, with more affinity for the MAP detector at small distances, since in this case the auxiliary channel assumed by the receiver is closer to the true channel (i.e., the effect of cascaded ROADMs is still not critical). In any case, simulations with LDPC codes confirm the reliability of the SE analysis performed through the AIR lower bounds computation. It is interesting to notice that our simulated rate- $4 / 5$ code with the SbS detector has the

\footnotetext{
${ }^{7}$ This reference BER of $10^{-4}$ has been clearly selected to reduce the simulation time. However, since these codes have a very steep waterfall, a BER below $10^{-7}$ can be achieved at a SNR value of just a fraction of $\mathrm{dB}$ higher. A much lower BER cannot be achieved with these codes since they have a an error floor around $10^{-7} \div 10^{-8}$. From this BER, additional outer codes with $2-3 \%$ overhead, tipically $\mathrm{BCH}$, can further reduce the BER down to $10^{-12}$ [9].
} 


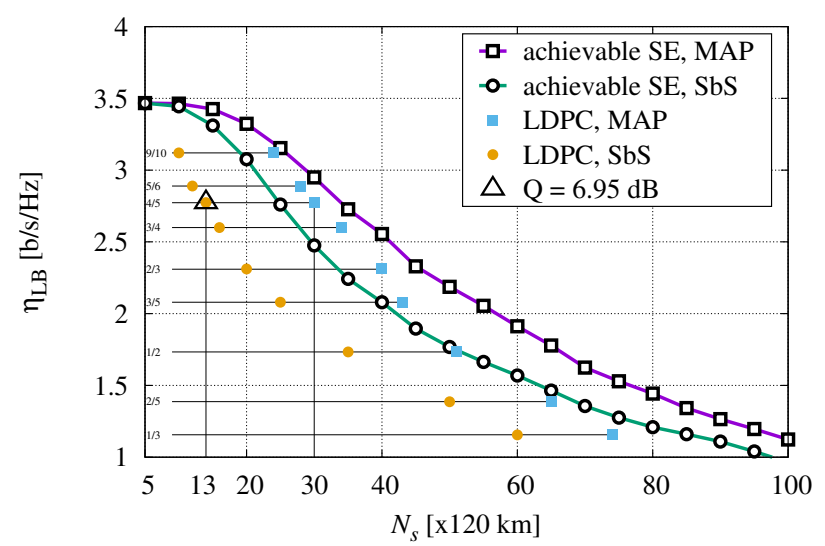

Figure 3. Spectral efficiency as a function of number of spans, at $2 \mathrm{dBm}$ launch power, $R=32.5$ Gbaud, $F=37.5 \mathrm{GHz}$, symbol-by-symbol ( $\mathrm{SbS}$ ) and MAP detectors, and simulations with rate $1 / 3,2 / 5,1 / 2,3 / 5,2 / 3,3 / 4,4 / 5,5 / 6$, 9/10 LDPC codes (with reference $\mathrm{BER}=10^{-4}$ after decoding).

same performance of the 6.95-dB reference code indicated by the triangle in Fig. 3, whereas if the same code is used with the MAP detector the reach can be more than doubled.

Finally, in Fig. 4 we compare the SE curves of Fig. 3 by defining the relative $\mathrm{SE}$ gain of the MAP receiver with respect to the $\mathrm{SbS}$ receiver as

$$
\Delta_{\eta_{\mathrm{LB}}}=\frac{\eta_{\mathrm{LB}, \mathrm{MAP}}-\eta_{\mathrm{LB}, \mathrm{SbS}}}{\eta_{\mathrm{LB}, \mathrm{SbS}}}
$$

where $\eta_{\mathrm{LB}, \mathrm{MAP}}$ and $\eta_{\mathrm{LB}, \mathrm{SbS}}$ are the achievable $\mathrm{SE}$ value of the MAP and symbol-by-symbol detector at each distance, respectively. It can be noticed that benefits are remarkable when the effect of WSS filtering starts to significantly impair the signal. Furthermore, the inset of Fig. 4 shows the gain of MAP detector in terms of distance reach

$$
\Delta_{N_{s}}=\frac{N_{s, \mathrm{MAP}}-N_{s, \mathrm{SbS}}}{N_{s, \mathrm{SbS}}},
$$

where $N_{s, \mathrm{MAP}}$ and $N_{s, \text { SbS }}$ represent the maximum reach for each detector with each code, versus achieved spectral efficiency. The gain decreases with increasing distance, as expected, but results show that the reach, up to the symbolby-symbol detector distance of $2400 \mathrm{~km}$, can be more than doubled if the 2-state MAP detector is employed.

\section{CONCLUSIONS}

In this paper, we investigated the effects of narrow filtering in WDM transmission over flexible grid optical networks, with particular emphasis on $37.5 \mathrm{GHz}$ spacing and $32.5 \mathrm{Gbaud}$ rate. We showed that it is possible to mitigate the detrimental intersymbol interference introduced by link cascaded WSS by employing a 2-state maximum-a-posteriori probability symbol detector for each input bit stream. The mitigation is quantified in terms of achievable spectral efficiency versus propagation distance and launch power. Moreover, we proved that it is possible to exploit LDPC codes of different rates in order to match the expected spectral efficiency, thus obtaining a remarkable system reach improvement, or, conversely, higher spectral efficiency at equal reach, with respect to the conventional symbol-by-symbol detector.

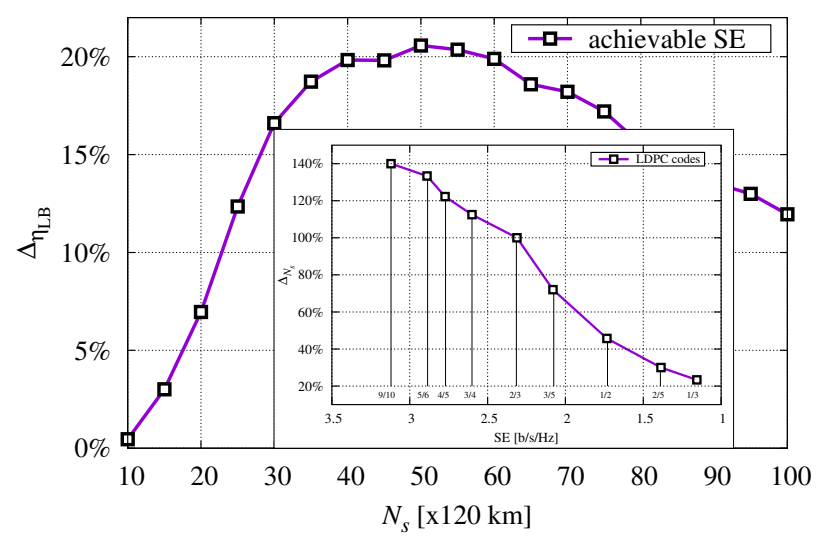

Figure 4. Relative spectral efficiency gain of MAP detector with $L=1$ with respect to conventional symbol-by-symbol detector, as a function of number of spans, at $2 \mathrm{dBm}$ launch power, $R=32.5 \mathrm{Gbaud}, F=37.5 \mathrm{GHz}$. The inset shows the gain in terms of distance reach $\Delta_{N_{s}}$ for each code in Fig. 3.

\section{ACKNOWLEDGMENT}

This work was supported by the Italian Ministero dell'Istruzione, dell'Università e della Ricerca (MIUR) under the FIRB project Coherent Terabit Optical Networks (COTONE).

\section{REFERENCES}

[1] G. Bosco, V. Curri, A. Carena, P. Poggiolini, and F. Forghieri, "On the performance of Nyquist-WDM terabit superchannels based on PMQPSK, PM-8PSK or PM-16QAM subcarriers," J. Lightw. Tech., vol. 29, no. 1, pp. 53-61, 2011.

[2] S. L. Jansen, B. Spinnler, I. Morita, S. Randel, and H. Tanaka, "100GbE: QPSK versus OFDM,” Optical Fiber Technology, vol. 15, pp. 407-413, Oct-Dec 2009

[3] R. Tkach and A. Chraplyvy, "Past/present system advances, with an eye towards the future," in Proc. European Conf. on Optical Commun. (ECOC'10), Sept. 2010.

[4] ITU-T G.694.1, "Spectral grids for WDM applications: DWDM frequency grid," Feb. 2012.

[5] Finisar, "Balancing performance, flexibility, and scalability in optical networks." White Paper, Feb. 2012.

[6] A. Morea, J. Renaudier, A. Ghazisaeidi, O. Bertrand-Pardo, and T. Zami, "Impact of reducing channel spacing from $50 \mathrm{GHz}$ to $37.5 \mathrm{GHz}$ in fully transparent meshed networks," in Proc. Optical Fiber Commun. Conf., (San Francisco, CA), 2014. paper Th1E.4.

[7] L. R. Bahl, J. Cocke, F. Jelinek, and J. Raviv, "Optimal decoding of linear codes for minimizing symbol error rate," IEEE Trans. Inform. Theory, vol. 20, pp. 284-287, Mar. 1974.

[8] G. Colavolpe and T. Foggi, "Time-frequency packing for high capacity coherent optical links," IEEE Trans. Commun., vol. 62, pp. 2986-2995, Aug. 2014.

[9] ETSI EN 301307 Digital Video Broadcasting (DVB); V1.1.2 (2006-06), "Second generation framing structure, channel coding and modulation systems for Broadcasting, Interactive Services, News Gathering and other Broadband satellite applications."

[10] J. Li, E. Tipsuwannakul, T. Eriksson, M. Karlsson, and P. A. Andrekson, "Approaching Nyquist limit in WDM systems by low-complexity receiver-side duobinary shaping," J. Lightw. Technol., vol. 30, pp. 16641676, June 2012.

[11] Z. Jia, Y. Cai, H.-C. Chien, and J. Yu, "Performance comparison of spectrum-narrowing equalizations with maximum likelihood sequence estimation and soft-decision output," Opt. Express, vol. 22, pp. 60476059, 2014.

[12] T. Rahman, A. Napoli, D. Rafique, B. Spinnler, M. Kuschnerov, I. Lobato, B. Clouet, M. Bohn, C. Okonkwo, and H. de Waardt, "On the mitigation of optical filtering penalties originating from ROADM cascade," IEEE Photon. Technol. Lett., vol. 26, pp. 154-157, Jan. 2014.

[13] N. Sambo, G. Meloni, F. Paolucci, F. Cugini, M. Secondini, F. Fresi, L. Potí, and P. Castoldi, "Programmable transponder, code and differentiated filter configuration in elastic optical networks," J. Lightw. Technol. vol. 32, pp. 2079-2086, June 2014. 
[14] O. V. Sinkin, R. Holzlöhner, J. Zweck, and C. R. Menyuk, "Optimization of the split-step fourier method in modeling optical-fiber communications systems," J. Lightw. Technol., vol. 21, pp. 61-68, Jan. 2003.

[15] G. Colavolpe, T. Foggi, E. Forestieri, and G. Prati, "Multilevel optical systems with MLSD receivers insensitive to GVD and PMD," J. Lightw. Tech., vol. 26, pp. 1263-1273, May 2008.

[16] G. Colavolpe, A. Barbieri, and G. Caire, "Algorithms for iterative decoding in the presence of strong phase noise," IEEE J. Select. Areas Commun., vol. 23, pp. 1748-1757, Sept. 2005.

[17] F. Rusek and A. Prlja, "Optimal channel shortening for MIMO and ISI channels," IEEE Trans. Wireless Commun., vol. 11, pp. 810-818, Feb. 2012.

[18] D. M. Arnold, H.-A. Loeliger, P. O. Vontobel, A. Kavčić, and W. Zeng, "Simulation-based computation of information rates for channels with memory," IEEE Trans. Inform. Theory, vol. 52, pp. 3498-3508, Aug. 2006.

[19] R. Essiambre and R. W. Tkach, "Capacity trends and limits of optica communication networks," Proc. IEEE, vol. 100, pp. 1035-1055, May 2012.

[20] R. Essiambre, G. Kramer, P. J. Winzer, G. J. Foschini, and B. Goebel, "Capacity limits of optical fiber networks," J. Lightw. Technol., vol. 28, pp. 662-701, Feb. 2010.

[21] N. Merhav, G. Kaplan, A. Lapidoth, and S. Shamai, "On information rates for mismatched decoders," IEEE Trans. Inform. Theory, vol. 40, pp. 1953-1967, Nov. 1994

[22] G. Colavolpe, T. Foggi, A. Modenini, and A. Piemontese, "Faster-thanNyquist and beyond: how to improve spectral efficiency by accepting interference," Opt. Express, vol. 19, pp. 26600-26609, Dec 2011.

[23] P. Serena, A. Bononi, and G. Colavolpe, "On the nonlinear capacity with memory of PS-QPSK and PDM-QPSK in WDM non-dispersion managed links," in Proc. European Conf. on Optical Commun. (ECOC'12), Sept. 2012. Paper We.2.C.1.

[24] G. Colavolpe and A. Barbieri, "On MAP symbol detection for ISI channels using the Ungerboeck observation model," IEEE Commun. Letters, vol. 9, pp. 720-722, Aug. 2005.

[25] U. Mengali and M. Morelli, "Data-aided frequency estimation for burst digital transmission," IEEE Trans. Commun., vol. 45, pp. 23-25, Jan. 1997.

[26] ITU-T G.675.1, "Forward error correction for high bit-rate DWDM submarine systems," Feb. 2004. 